\title{
RESULTS OF A 25-YEAR SCREENING PROGRAMME FOR NEONATAL HIP INSTABILITY
}

\author{
M. F. MACNICOL
}

From the Princess Margaret Rose Orthopaedic Hospital, Edinburgh

\begin{abstract}
From 1962 to 1986, 117256 neonates were screened for congenital dislocation of the hip (CDH). When the primary physical examination was performed by the junior paediatric staff there was a persistent late diagnosis rate of 0.5 per 1000 live births.

When the primary examination was undertaken by experienced orthopaedic personnel (1982 to 1984) the late diagnosis rate fell and fewer infants were splinted.
\end{abstract}

Examination of the hips is included as part of the routine examination of newborn infants, although its value is not universally established (MacKenzie and Wilson 1981; Catford, Bennet and Wilkinson 1982). There is no doubt that splinting the neonatal hip in abduction diminishes the risk of congenital dislocation (von Rosen 1962; Mitchell 1972; Fredensborg 1976; Palmén 1984; Dunn et al 1985; Hadlow 1988), but the problems of recruiting experienced personnel for screening programmes and of maintaining their enthusiastic vigilance, are considerable, particularly if the poor prognosis of the missed dislocation is not constantly reiterated.

A screening programme was established in the major maternity unit of Edinburgh in 1962. This paper reports the results of this service and of a subsidiary prospective trial in which two experienced members of the orthopaedic department undertook the primary examinations from 1982 to 1984.

\section{PATIENTS}

Over the 25-year period from 1962 to 1986,117256 infants were examined for hip instability at birth (Fig. 1). The initial examination was by one of the paediatric junior staff, all of whom had been instructed in the technique of examination by an orthopaedic surgeon. In addition to the Ortolani and Barlow tests (Barlow 1962),

M. F. Macnicol, BSc (Hons), MCh, FRCS Ed(Orth), Consultant Orthopaedic Surgeon and Senior Lecturer

Princess Margaret Rose Orthopaedic Hospital, 41-43 Frogston Road West, Edinburgh EH10 7ED, Scotland.

(C) 1990 British Editorial Society of Bone and Joint Surgery

$0301-620 \mathrm{X} / 90 / 6030 \$ 2.00$

J Bone Joint Surg [Br] 1990; 72-B : 1057-60 they were instructed to look for skeletal asymmetry, limitation of abduction, and loss of the physiological flexion deformity of the hip found in the normal neonate. The family history and the circumstances of the pregnancy and delivery were noted. All infants were reexamined before their discharge home. Babies with obviously unstable hips and those which caused suspicion were referred for further examination by an experienced member of the orthopaedic department and a standardised radiograph of the pelvis was taken (Bertol, Macnicol and Mitchell 1982).

From 1962 to 1968 , the hips were treated with the Malmö splint, not only if they were found to be abnormal by the orthopaedic surgeon, but also if they had been reported unstable at birth and later stabilised, particularly if the pelvic radiograph was abnormal. This latter group

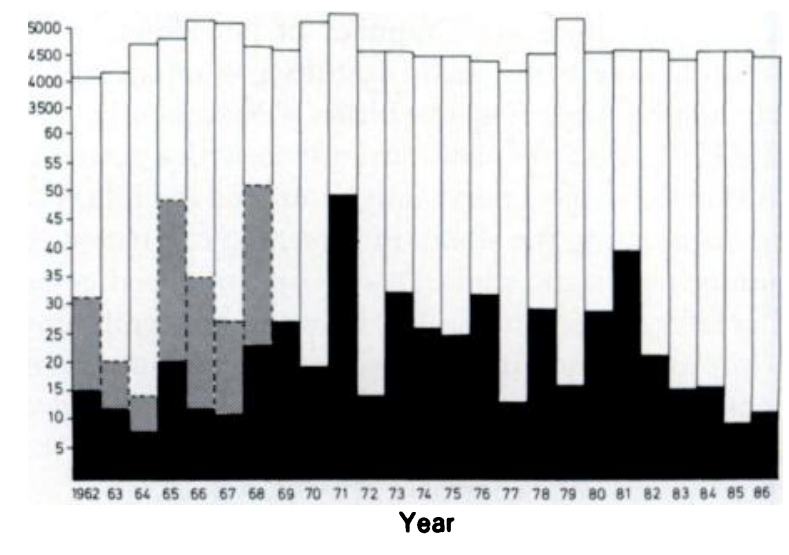

Fig. 1

Annual numbers of live births and of cases splinted, 1962 to 1986; $\square$ live births; $\square$ hips splinted for instability; $\mathbb{Z}$ hips splinted for minor instability (see text). 
of patients, with minor instability, is shown by the hatched areas in the bar graph in Figure 1.

From 1969 to 1986 only those babies with instability of the hip confirmed by the orthopaedic examiner were splinted. We used the Malmö splint initially, and the Pavlik harness more recently. If the first examination suggested that the hip was abnormal, but subsequent examination provided no cause for concern, the infant was not treated, though such cases were reviewed at a special clinic for CDH at the orthopaedic hospital.

Further examinations of the hips are undertaken by community medical staff when the child is six weeks and 10 months of age.

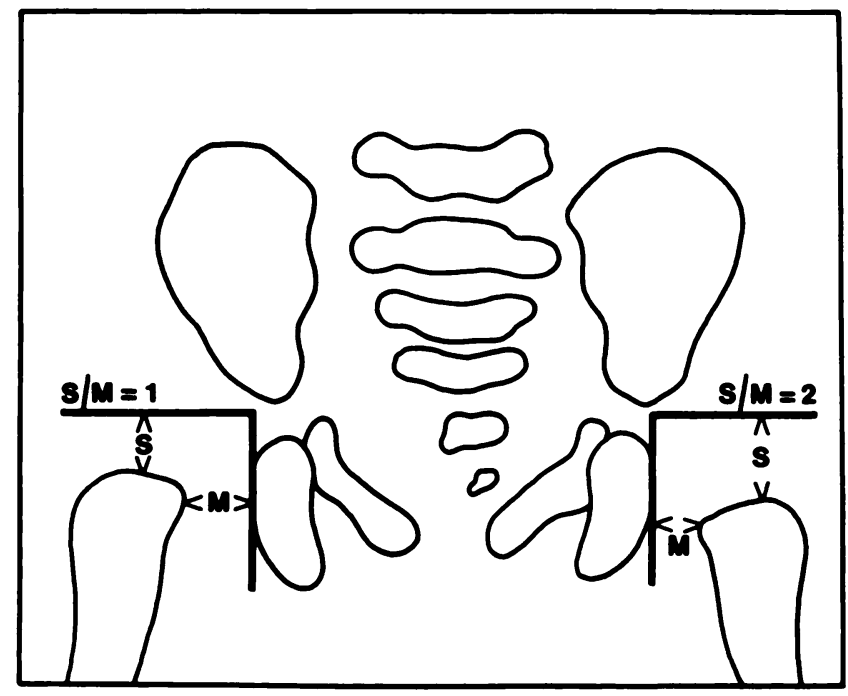

Fig. 2

Method of measuring radiographs. The medial gap $(M)$ and superior gap (S) are measured in millimetres. In the stable hip the ratio $\mathbf{S} / \mathbf{M}=$ 2. Progressive instability produces a ratio that approximates to 1 .

Prospective study (1982 to 1984). In early 1982 it became clear that an appreciable number of late diagnoses of dislocation were being made despite a splintage rate of approximately 6 per 1000 live births. Consequently, from 1982 to 1984 , two orthopaedic examiners with a particular interest in the subject personally examined the infants at birth, augmenting the standard screening carried out by the junior paediatric staff. This prospective trial generated greater interest about $\mathrm{CDH}$ in the maternity unit, and improved the links between the paediatric and orthopaedic staff. Radiographs were still requested as before, using a modified method based upon that previously described (Bertol et al 1982, Fig. 2).

At the end of this trial, paediatric staff were left to conduct their own neonatal hip assessments without formal supervision. Infants with hips that they considered to be abnormal were still reviewed in the maternity unit on request, with splintage and follow-up arranged by the orthopaedic staff as before. Out-patient examination of infants by community care doctors during the first year of life was continued, thus ensuring that secondary screening would identify abnormal hips before walking age (Palmén 1984).

\section{RESULTS}

Approximately 4500 infants were examined annually during the period of the screening programme. Splintage was recommended in 652 cases, $28 \%$ for bilateral instability, $51 \%$ for left-sided instability, and $21 \%$ for right-sided instability. The number of cases splinted has

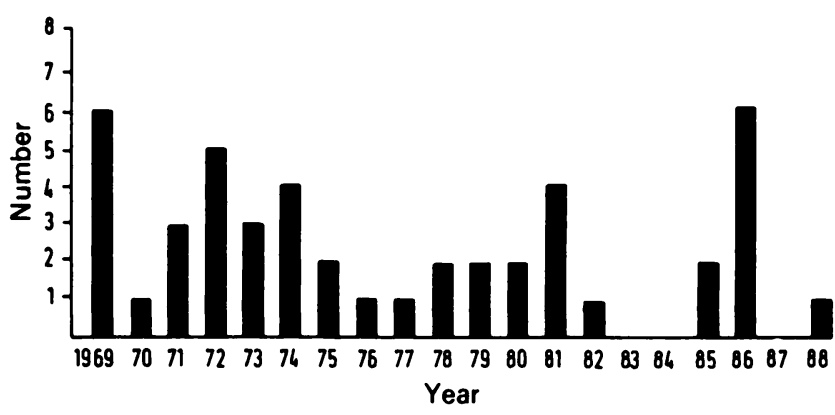

Fig. 3

Variations in the number of late diagnoses over the last 20 years (see text).

gradually reduced from more than 6 per 1000 to under 3 per 1000. This low splintage rate became established during the time of the prospective study (1982 to 1984) when we became more confident about which doubtful hips needed only to be reviewed in the $\mathrm{CDH}$ clinic. If formal splintage was thought unnecessary but the hips were not considered to be entirely normal, the infant was nursed prone with legs abducted. The splintage rate did not change when the paediatrician took over the primary examination themselves in 1985; all babies with suspicious hips were thereafter referred to the orthopaedic staff.

Figure 3 clearly shows the impact of the two experienced examiners upon the late diagnosis rate in 1983 and 1984 . The secondary, community-based screening ensured that these late cases were diagnosed within the first year of life in all but one instance.

Five years have elapsed since the completion of the prospective trial and it is unlikely that any late cases will now present. The only case missed during that period was in early 1982 and it was diagnosed at a six-week examination. A late diagnosis rate of 0.5 per 1000 live births during the first 20 years of the screening programme (two or three missed cases annually) was therefore reduced to virtually nil by the introduction of experienced examiners. The splintage rate was also reduced since 
there was less diagnostic uncertainty, although a policy of reviewing all suspicious cases was pursued by referring doubtful cases to the $\mathrm{CDH}$ clinic.

The alarming rise in the late diagnosis rate in 1985 and 1986 (Fig. 3) was halted by the re-introduction of experienced orthopaedic personnel who are now available during the time of the primary neonatal examinations and consult with the junior paediatric staff on a daily basis. Ideally, this commitment is undertaken by a trained medical assistant, a physiotherapist, or a nurse who attends both the maternity unit and the $\mathrm{CDH}$ clinic.

\section{COMPLICATIONS}

The incidence of dislocation after splintage is very low. In seven instances the Malmö splint failed to control the hips (Fig. 4), and in three the Pavlik harness proved inadequate, but stability was achieved by changing to the Malmö splint. In two of the latter cases maternal compliance was poor and the splint was probably not worn regularly.
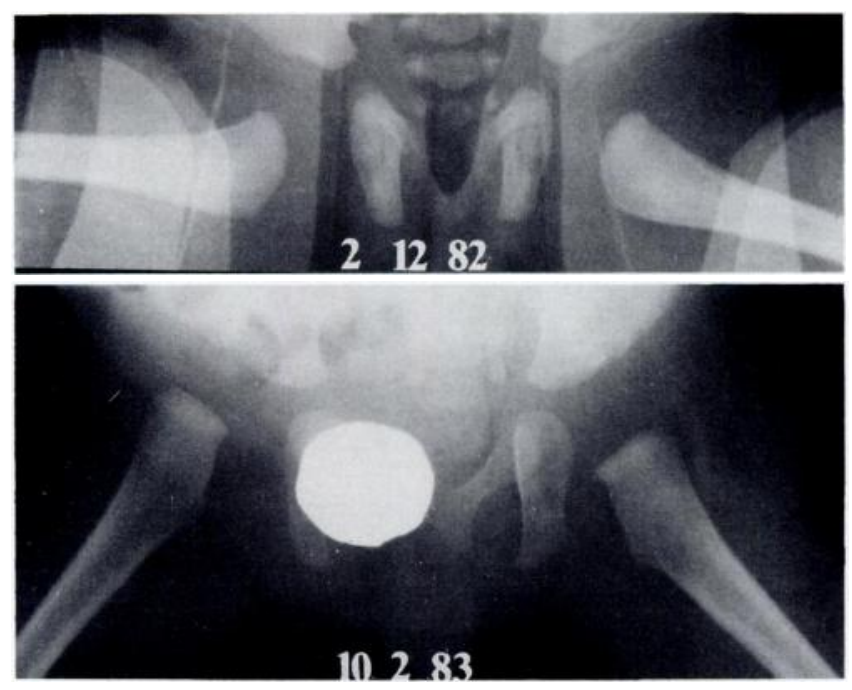

Fig. 4

Although the Malmö splint apparently reduced the hips well (above), the right hip subsequently dislocated (below). A soft-tissue obstruction was later defined arthrographically.

Splintage is advised for a minimum of 12 weeks, although it is appreciated that a shorter period may suffice. Two infants with very unstable hips were managed briefly with light plaster spicas, but the risks of producing proximal femoral ischaemia are so great at this age that treatment should usually be deferred or preceded by arthrography in order to define any softtissue obstruction.

Some delay in proximal femoral epiphyseal ossification is seen radiographically in all unstable hips (Bertol et al 1982; Hadlow 1988), but the appearances usually become symmetrical before nine months of age. Transient irregularity of ossification persisted in $3 \%$ of our splinted cases, but with no eventual abnormality of femoral head development. Avascular necrosis affected the femoral head in six early cases, when the Malmö splint was applied in too much abduction; three of these resulted in partial growth arrest.

\section{DISCUSSION}

We have shown that careful examination of the neonatal hip by experienced personnel can significantly reduce the late diagnosis rate of $\mathrm{CDH}$. There are risks and disappointments associated with neonatal screening (MacKenzie and Wilson 1981; Moore 1989), but the recruitment and training of nurses or physiotherapists is recommended, provided that a close association with the local orthopaedic service is established. The cost to the community of each dislocation diagnosed late, whether in the expense of later operative treatment and morbidity or in medicolegal compensation, would provide an appropriate salary for such a clinical assistant.

Careful physical examination may also make it less compelling to introduce more costly and time-consuming investigations such as ultrasound (Clarke, Clegg and AlChalabi 1989) and vibration emission (Cowie et al 1983). The value of these techniques in reducing the late diagnosis rate has not yet been proved and all such adjuncts to diagnosis, including radiography, rely upon the discrimination afforded by the initial physical examination. This basic test still decides whether clinical suspicion about instability is warranted.

Reliance upon screening of infants later, in the first year of life, may also be ill-founded since skewing of the pelvis (Macnicol 1987) and radiographic variations in the ossification of the femoral head and acetabulum may lead to indecision about when to splint and when to observe. However, Palmén (1984) has made a convincing case for further examination of all infants during the first year of life, to augment the primary screening procedure. In Edinburgh, this policy succeeded in lowering the age at which late diagnoses were made from an average of 17 months to eight months.

In conclusion, careful physical examination of the neonate for the signs of hip instability can reduce the late diagnosis rate substantially, provided it is done by experienced personnel. If a maternity unit has trained staff available to examine all newborn infants and re-test or splint those with abnormal hips within the first two or three days after birth, then it is possible virtually to eradicate late-diagnosed cases of hip instability. The incidence of splintage should also be reduced.

I wish to thank Dr M. Moonsawmy and the paediatric junior staff of the Simpson Memorial Maternity Pavilion, Royal Infirmary of Edinburgh.

No benefits in any form have been received or will be received from a commercial party related directly or indirectly to the subject of this article. 


\section{REFERENCES}

Barlow TG. Early diagnosis and treatment of congenital dislocation of the hip. J Bone Joint Surg [Br] 1962; 44-B:292-301.

Bertol P, Macnicol MF, Mitchell GP. Radiographic features of neonatal congenital dislocation of the hip. J Bone Joint Surg [Br] 1982; 64B:176-9.

Catford JC, Bennet GC, Wilkinson JA. Congenital hip dislocation: an increasing and still uncontrolled disability? Br Med J 1982; 285:1527-30.

Clarke NMP, Clegg J, Al-Chalabi AN. Ultrasound screening of hips at risk for $\mathrm{CDH}$ : failure to reduce the incidence of late cases. $J$ Bone Joint Surg [Br] 1989; 71-B :9-12.

Cowie GH, Bogues BA, Kerohan WG, Mollan RAB. A new aid in diagnosis of congenital dislocation of the hip. $J$ Bone Joint Surg [Br] 1983; 65-B:656.

Dunn PM, Evans RE, Thearle MJ, Griffiths HED, Witherow PJ. Congenital dislocation of the hip: early and late diagnosis and management compared. Arch Dis Child 1985; 60:407-14.

Fredensborg $\mathbf{N}$. The results of early treatment of typical congenital dislocation of the hip in Malmö. J Bone Joint Surg [Br] 1976; 58B :272-8.
Hadlow V. Neonatal screening for congenital dislocation of the hip: a prospective 21-year survey. J Bone Joint Surg [Br] 1988; 70-B 740-3.

MacKenzie IG, Wilson JG. Problems encountered in the early diagnosis and management of congenital dislocation of the hip. J Bone Joint Surg [Br] 1981 ; 63-B :38-42.

Macnicol MF. Congenital dislocation of the hip. In: Bennet GC, ed. Paediatric hip disorders. Oxford, etc: Blackwell Scientific Publications, 1987:64-113.

Mitchell GP. Problems in the early diagnosis and management of congenital dislocation of the hip. J Bone Joint Surg [Br] 1972; 54B:4-12.

Moore FH. Examining infants' hips - can it do harm? J Bone Joint Surg [Br] 1989; 71-B:4-5

Palmén K. Prevention of congenital dislocation of the hip: the Swedish experience of neonatal treatment of hip joint instability. Acta Orthop Scand 1984; Suppl 208; 55:1-107.

von Rosen S. Diagnosis and treatment of congenital dislocation of the hip in the new-born. J Bone Joint Surg [Br] 1962; 44-B: 284-91. 